\title{
The Critical Stable Length in Wrinkles of Two-Dimensional Materials
}

\section{AUTHOR NAMES}

Fangyuan Zheng ${ }^{1,2+}$, Quoc Huy Thi ${ }^{3,4+}$, Lok Wing Wong ${ }^{1,2}$, Qingming Deng ${ }^{5 *}$, Thuc Hue Ly ${ }^{3,4} *$ and Jiong Zhao ${ }^{1,2} *$

\section{AUTHOR ADDRESS}

1 Department of Applied Physics, The Hong Kong Polytechnic University, Kowloon, Hong Kong, China.

${ }^{2}$ The Hong Kong Polytechnic University Shenzhen Research Institute, Shenzhen 518000, China.

3 Department of Chemistry and Center of Super-Diamond \& Advanced Films (COSDAF), City University of Hong Kong, Kowloon, Hong Kong, China.

${ }^{4}$ City University of Hong Kong Shenzhen Research Institute, Shenzhen 518000, China.

5 Physics department and Jiangsu Key Lsituoratory for Chemistry of Low-Dimensional Materials, Huaiyin Normal University, Huaian 223300, China.

* emails: qingmingdeng@gmail.com, thuchly@,cityu.edu.hk, jiongzhao@polyu.edu.hk

+ These authors contribute equally.

The authors declare no competing financial interest. 
$v d W$ force in bended/rippled $2 D$ materials. A previous study reported the critical states of collapsed graphene wrinkles, which shows that there are two states before the wrinkles are folded: one is the state of rippling before two sidewalls attracted by the $\mathrm{vdW}$ force, and the other is the standing and collapsed wrinkle. In our experiment, the vdW force is a key parameter that determines the shapes of the wrinkle during the continuous change. For the formation process of each wrinkle, in the beginning, the two sides of the wrinkle peaks are not close enough, which can be regarded as no/weak vdW interactions on the two monolayers. With the movement of the W tip, the two sides become increasingly closer, and the $\mathrm{vdW}$ force provides an attractive force to the two-side bended layers and induces the formation of a collapsed wrinkle. Afterwards, when the length continues increasing, the morphologies of the curved top part do not change, as the height of the wrinkle increases.

Measurement of the wrinkle length and error analysis. Form the captured video images of the samples, the carbon film is in ellipse but not circle. This shape is caused by the inclination of the sample for better contact with the $\mathrm{W}$ tip and the distortion of the carbon film under the force of the $\mathrm{W}$ tip. The value of the distortion can be calculated by the ratio of the major and minor axes of the ellipse, and obviously, the length of the wrinkle is along the minor axis. We need to take the distortion value into account during the measurement.

In addition, during the measurement, we notice that the moving direction of the $\mathrm{W}$ tip between each pair of frames is not exactly the same due to the rotation of ripples and the Nanofactory controller. To obtain the precise values, the movement of 5 different spots on the wrinkle is measured each time and the average value is obtained. Additionally, the direction of wrinkles slightly changes from one direction to another. Furthermore, the distance of the two adjacent wrinkles is different from left to right, which 
causes a difference in the friction. Here, we also take the average values of several spots on the wrinkles. Other factors, such as the contamination on the sample and the influence of the electron beam, which can lead to errors, are minimized by the dry transfer method, low magnification and low electron dose observation of TEM, respectively.

Quantitative calculation on the frictional force. From Eq.1 we measured the value of $\Delta l$ and $x$ from TEM experiment. Eq.1 is to explain the friction force is related to the area of contact, which is consistent with the result of measurement.

In our TEM experiment, we approximate the contact area in front of each wrinkle is a rectangle, so the friction force is linearly dependent on the contact area. The interlayer friction coefficient value is 2.60 pN/atom among the multilayer graphene sample. From Fig. 3b, given a certain value of $x$ which is measured in our experiment, we can calculate the value of the friction force. For example, when $x=22.9$ $\mathrm{nm}$ in the first snapshot, $f=\mu x=2.60 \mathrm{pN} *(22.9 / 0.14)=0.43 \mathrm{nN}$. Meanwhile, according to the dimension of our W STM tip (around $6 \mathrm{~nm}$ diameter and $100 \mathrm{~nm}$ length), the force constant $\mathrm{k}$ of the STM tip is estimated by one fixed-end beam deflection model to be $0.04 \mathrm{nN} / \mathrm{nm}$, and the friction force by $f=k \Delta l$ yielded $0.04 * 9=0.36 \mathrm{nN}$, close to the $0.43 \mathrm{nN}$ above.

Continuum mechanics model for the wrinkles. The model reconstructs the wrinkle in each type under different values of $\gamma$. In each case, the length of the wrinkle and energy can be calculated by the given parameters. To determine the critical state with and without vdW forces, the length and energy are similar. With an increase in $\gamma$ excluding $\mathrm{vdW}$, at $l=4.046 \mathrm{~nm}$, the two sides are attracted together, and the energy is $20.665 * 10^{-10} \mathrm{~J} / \mathrm{nm}$. Then, with an increase of 2 atoms, which is $0.24 \mathrm{~nm}$, the vdW 
monolayer wall is formed, containing an energy of $21.619 * 10^{-10} \mathrm{~J} / \mathrm{nm}$. The energy can be regarded as the same within the margin of error.

As mentioned above, the value of $\gamma$ largely depends on the bending stiffness and the adhesion energy between monolayers. The values of $W_{a d}$ and $D$ in monolayer graphene are variable in previous experiments and calculations, and in our case, we use the value of $\operatorname{sp} 2$ carbon with $D=1870 \mathrm{meV}$ and $W_{a d}=14 \mathrm{meV} / \mathrm{A}^{2}$, which should be consistent with the experimental value of monolayer graphene. Nevertheless, there may be a slight change in the critical state and the value of energy due to the change in $W_{a d}$ or $D$.

Comparison of the monolayer on the substrate and freestanding monolayer. In our modelling, the value of $W_{a d}, f$ and the energy calculation are apparently determined by the materials and contact of the bottom. For monolayer graphene with the graphene substrate, it is not difficult to find the related parameters from the results of experiments and calculations. However, it can be complicated when deciding the parameters in a freestanding monolayer. More calculation and dynamical tests are needed to obtain an accurate value of $W_{a d}$ since the supporting force is derived from the unpeeled part connected with the whole membrane and the carbon film under the sample. 


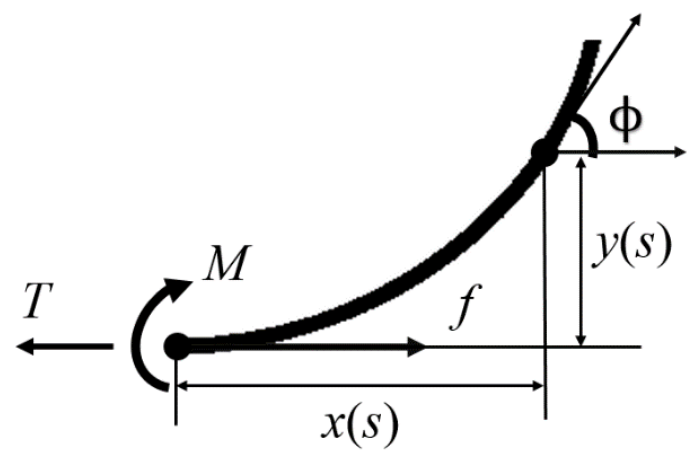

From the equations in the Methods section, the boundary conditions decide the shape of wrinkles in the different cases during modelling. During the exfoliation and recovery process of monolayer graphene in the main text, the movement of the wrinkle is controlled by the $\mathrm{W}$ tip. For the curvature of the wrinkle, one side is connected with the whole graphene flake so it always starts with the boundary condition $\phi(0)=0$, as well as $x(0)=0, y(0)=0$. Another boundary condition is $\phi^{\prime}(0)=\sqrt{\frac{W_{a d}}{D}}$, as a result of J-integral, stationary potential and material force balance. From the normalization of $x, y, s$, and $\phi$, the curvature of wrinkles under different values of $f$ (friction force) can be determined, which corresponds to the wrinkle state under the force applied by the W tip. With regard to the other case in Figure 5 (main text), in freestanding $\operatorname{ReS}_{2}$ introduced in the main text, there is a slight change in the boundary conditions. Both sides of the edge are flexible and can move, so $x(0)=0$ is unsuitable. The way in which the $\mathrm{W}$ tip contacts the sample is not from the bottom but from the side, so the end connected with the W tip cannot reach $\phi(0)=0$ and the curvature of the first wrinkle is less than a whole period. Meanwhile, there is no friction force between the wrinkles and substrate due to the dangling ripple. Nevertheless, additional dynamical tests are needed to determine the mechanical properties of these unknown materials in further studies. 


\section{Supporting Figures}
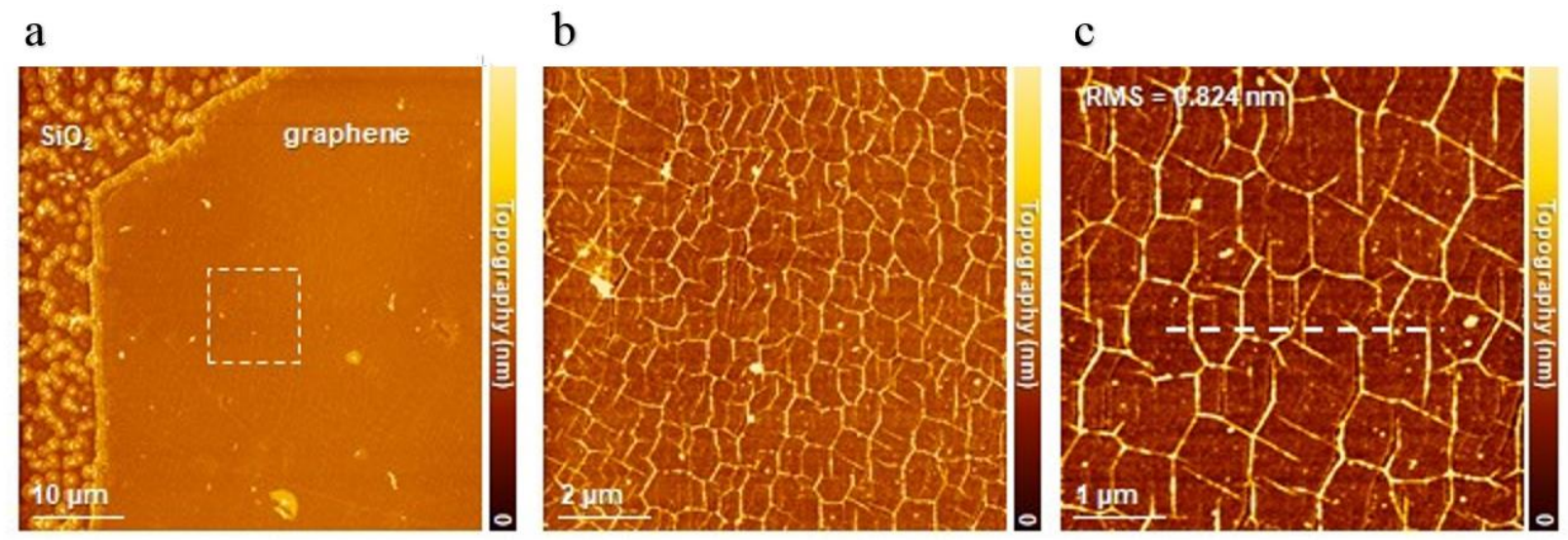

Figure S1। (a)-(c), The wrinkles formed on $\mathrm{SiO}_{2}$ after transfer, AFM topographic images with three magnifications for the same area (white dashed rectangle). 

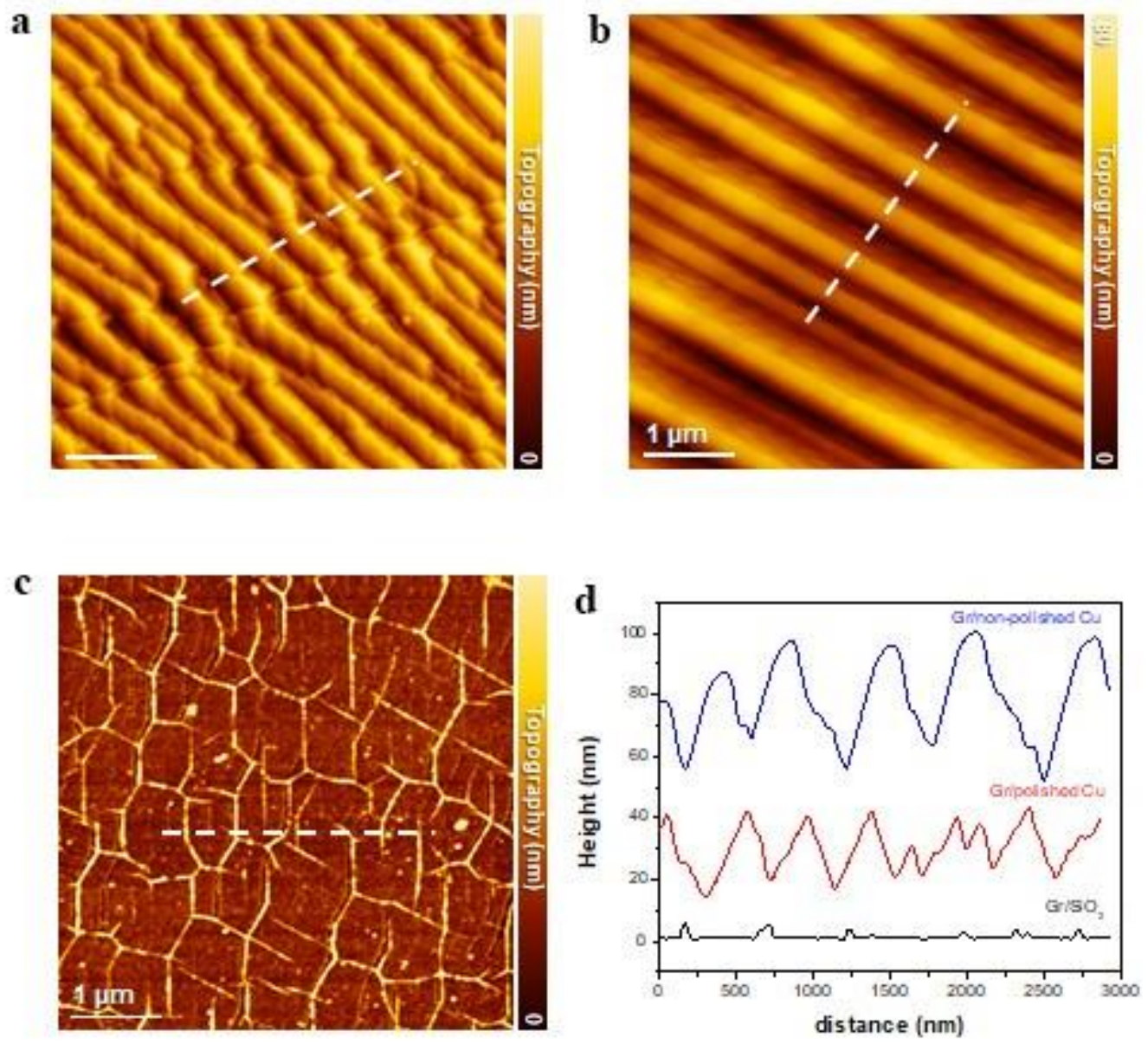

Figure S2। (a)-(c), The AFM topographic image for the $1 \mathrm{~L}$ graphene on polished $\mathrm{Cu}, 1 \mathrm{~L}$ graphene on non-polished $\mathrm{Cu}$, and $1 \mathrm{~L}$ graphene on transferred $\mathrm{SiO}_{2}$ substrate. (d), The height profiles corresponding to the white dashed lines in (a)-(c). The wrinkles formed after transfer are much smaller than the original terraces/steps of $\mathrm{Cu}$ foils. The smaller wrinkles after transfer does not follow the direction of any surface steps, instead, they follow the graphene lattices, zigzag or armchair directions (two energy favorable directions for wrinkle formation), therefore, the angles between wrinkles in panel (c) are $30^{\circ}, 60^{\circ}, 90^{\circ}, 120^{\circ}$ and $150^{\circ}$. The graphene growth on polished $\mathrm{Cu}$ foil still have parallel and sparse wrinkles because of the differential thermal expansion coefficient between graphene and $\mathrm{Cu}$ foil, these wrinkles mainly follow the surface terraces/steps of the $\mathrm{Cu}$, and they are much higher (10 50 nm height) than the lower wrinkles (1 5nm height) 
formed by PMMA transfer on the $\mathrm{Si} / \mathrm{SiO}_{2}$ substrate. 

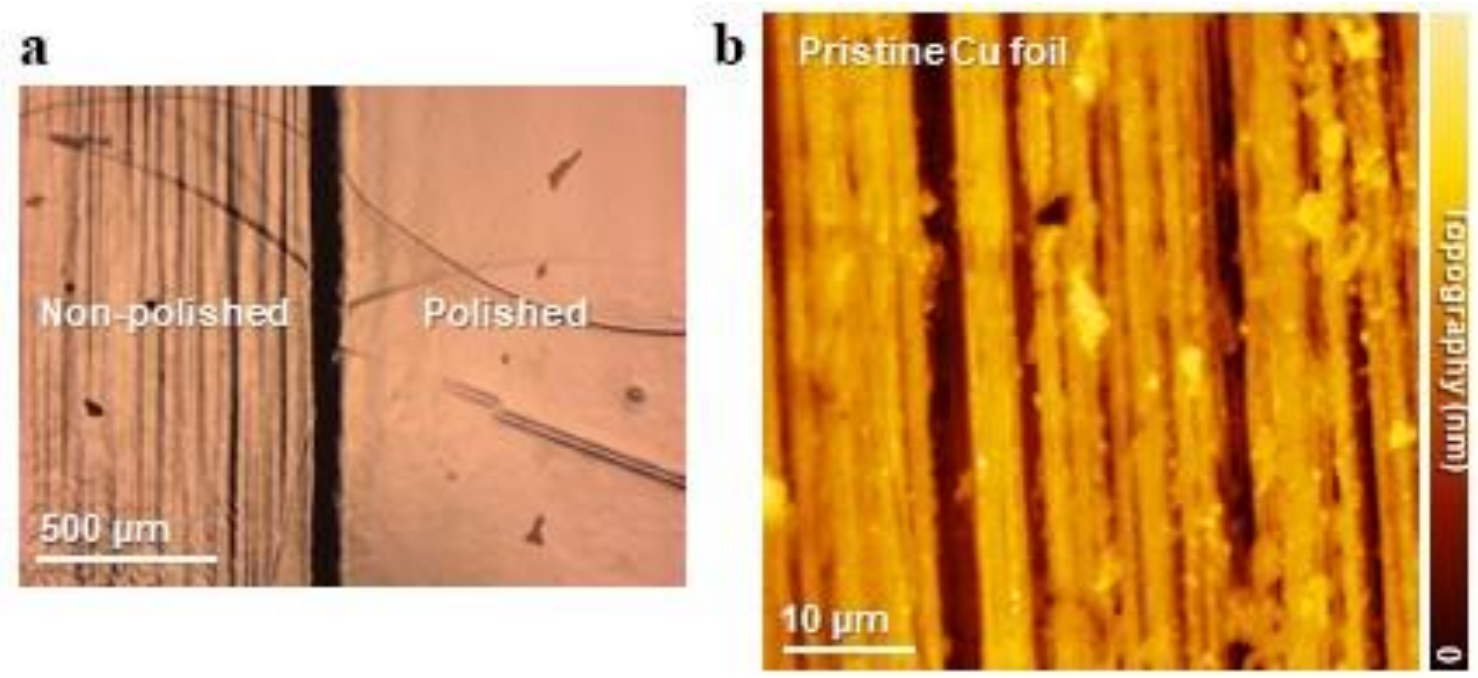

Figure S3। (a), OM image of the monolayer graphene grown on partially polished $\mathrm{Cu}$ foil. (b), The AFM topographic image for the pristine $\mathrm{Cu}$ foil with parallel steps. The parallel wrinkles/patterns (as in panel (b) in above figure) on pristine $\mathrm{Cu}$ foil are reduced after polishing. 

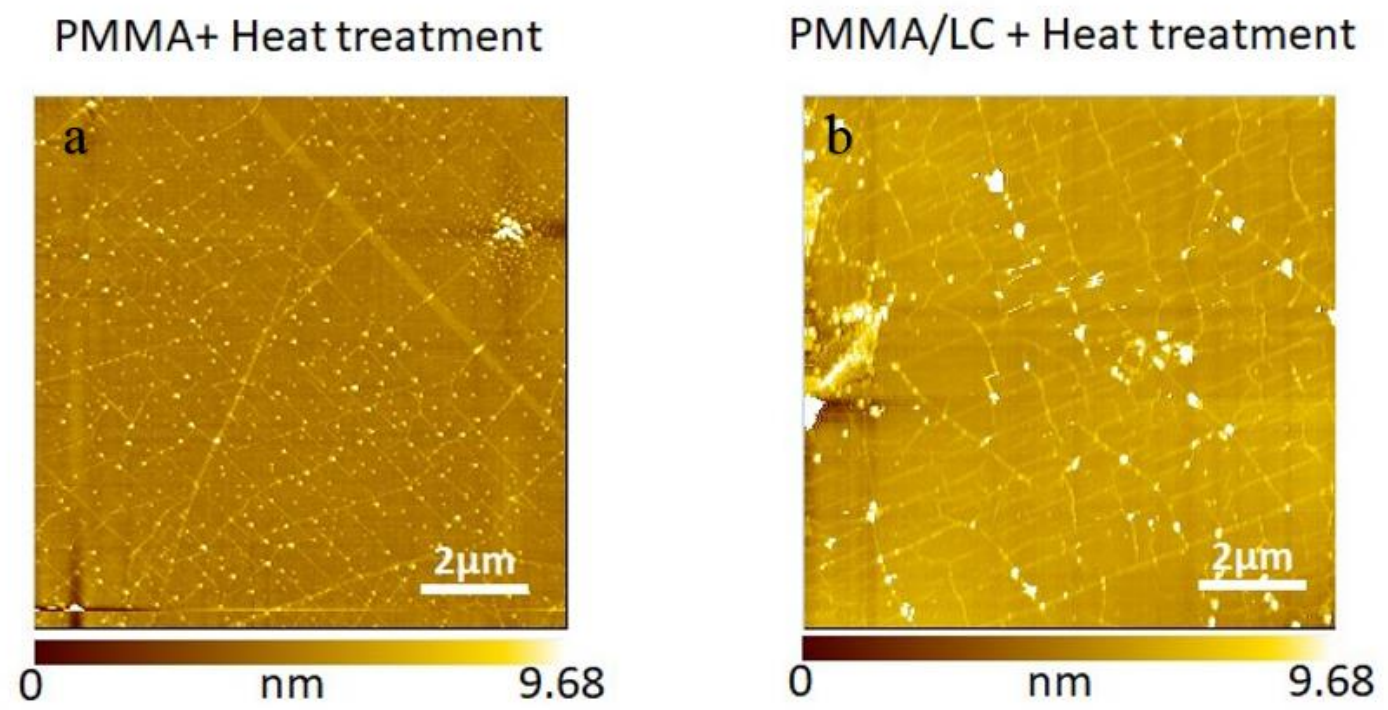

Figure S4| (a)AFM topographic images for the samples with normal PMMA transfer approach with thermal annealing. (b), liquid crystal (LC)/PMMA transfer approach with thermal annealing. The hillock and articles (residues) are significantly reduced by using liquid crystals during transfer. 

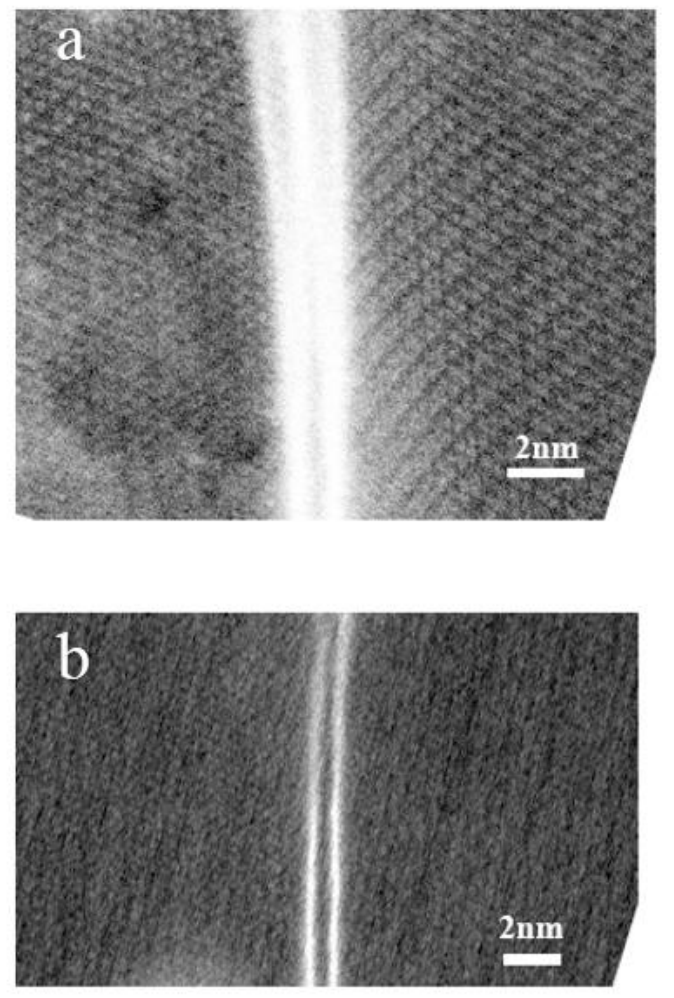

Figure S5। (a), (b)The high resolution HAADF STEM images (plane-view) for the winkles in (suspended) 2D ReS 2 , showing much smaller widths than in AFM images which have significant widening effect. 

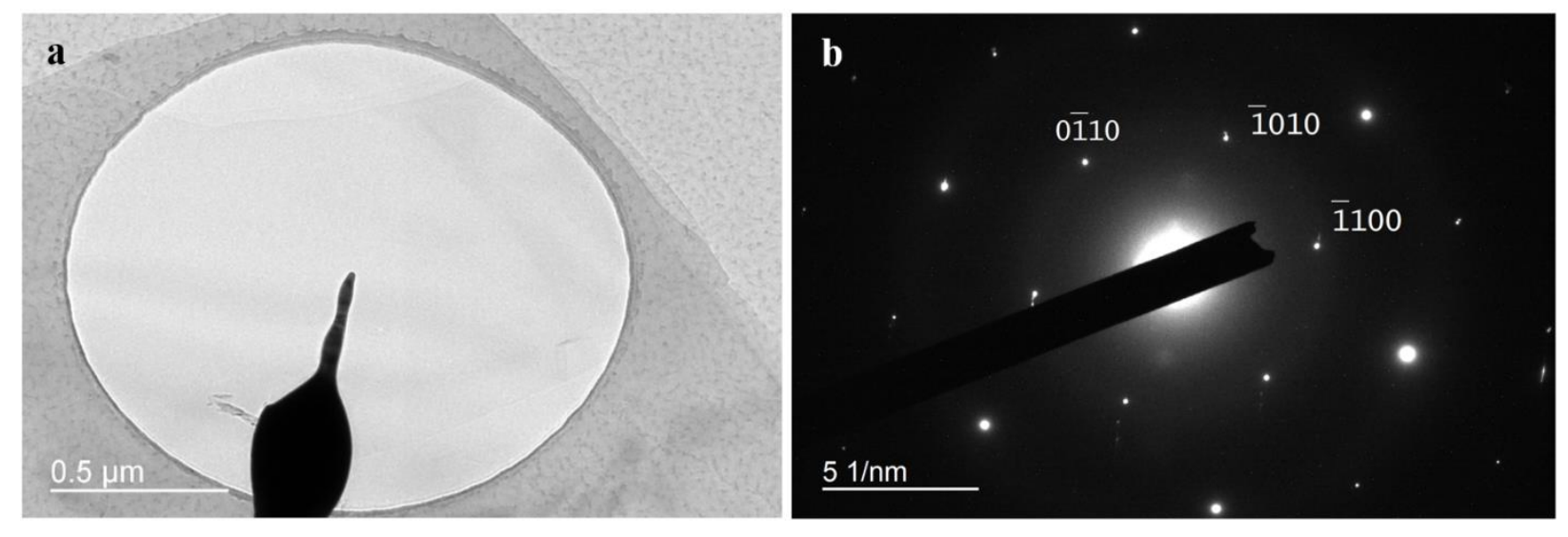

Figure S6। (a) The multilayer graphene flake covering the carbon film under magnification of 10k. (b) SAED pattern of multilayer graphene flakes. The cracking plane is along the [-1100] crystal direction. 
a
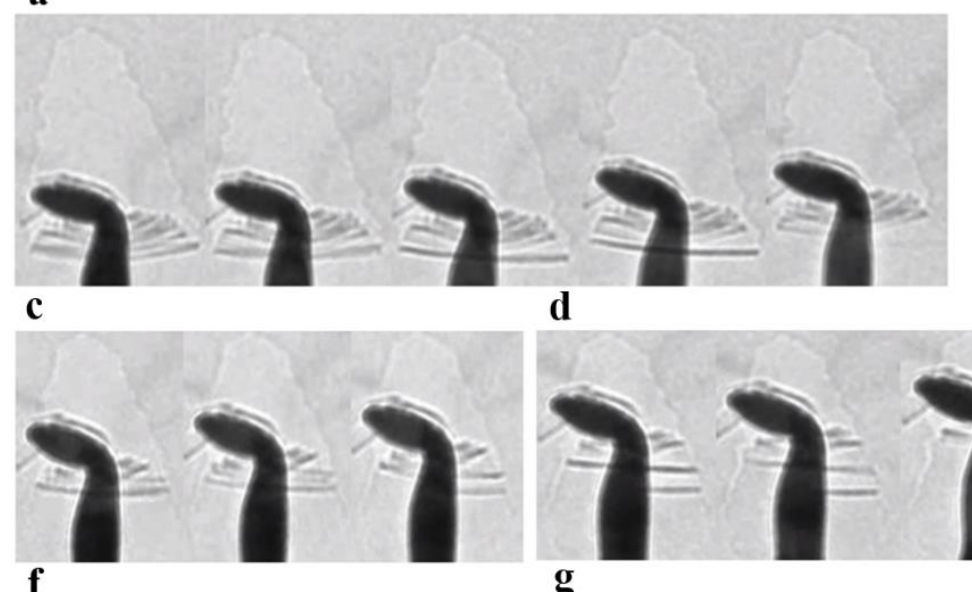

f

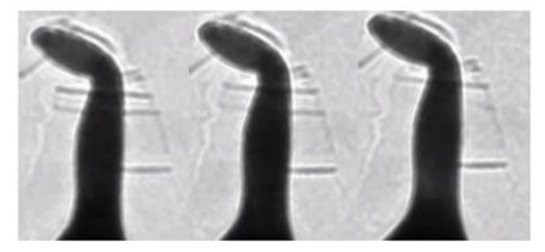

b

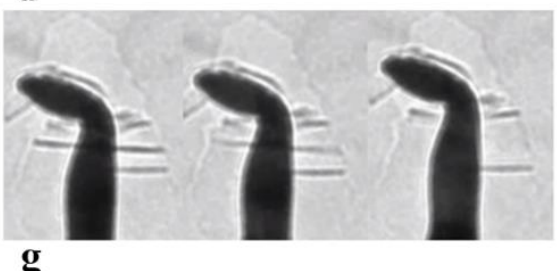

g

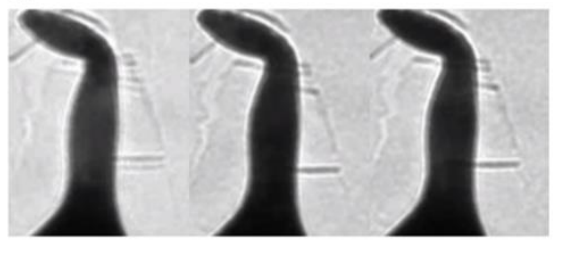

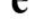

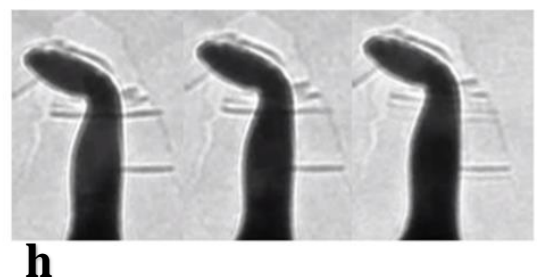

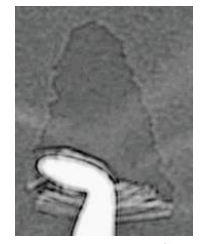

Figure S7| The recovery process of wrinkles 1-7 (a)-(g) when the W tip moves upward. During the recovery process, each wrinkle always starts from the formation of a full wrinkle with dark contrast until the wrinkle becomes narrow and disappears. The wrinkles in (a) and (b) can be regarded as straight wrinkles with tiny rotation between the top layer and the bottom wrinkles, which can be neglected. In (c), after recovery, there is a small wrinkle formed on the right part leading to the rotation of the top layer with wrinkles afterwards. Similar process also occurs in the process of (f), and the two small wrinkles remain on the ripple until the end of the entire process. In the end, almost all the single wrinkles are gone, and only several small folds remain near the edge due to a distortion of the ripple caused by the $\mathrm{W}$ tip. Thus, the relative length of the sheet is the distance of the previous wrinkle moved from the formation to the disappearance of the bottom one. The force applied by the $\mathrm{W}$ tip can be decomposed into two parts: one part overcomes the friction between the monolayer graphene sheet and the substrate, and the other 
part creates an extra movement on the wrinkles. In the TEM images, the length of the wrinkle is the distance of how many previous wrinkles can be moved on the substrate and the relative movement of the bottom wrinkle to the second bottom one. (h), The contrast-enhanced TEM figure. All the crack edges are single lines, which is a proof that the ripple been peeled off is monolayer. 

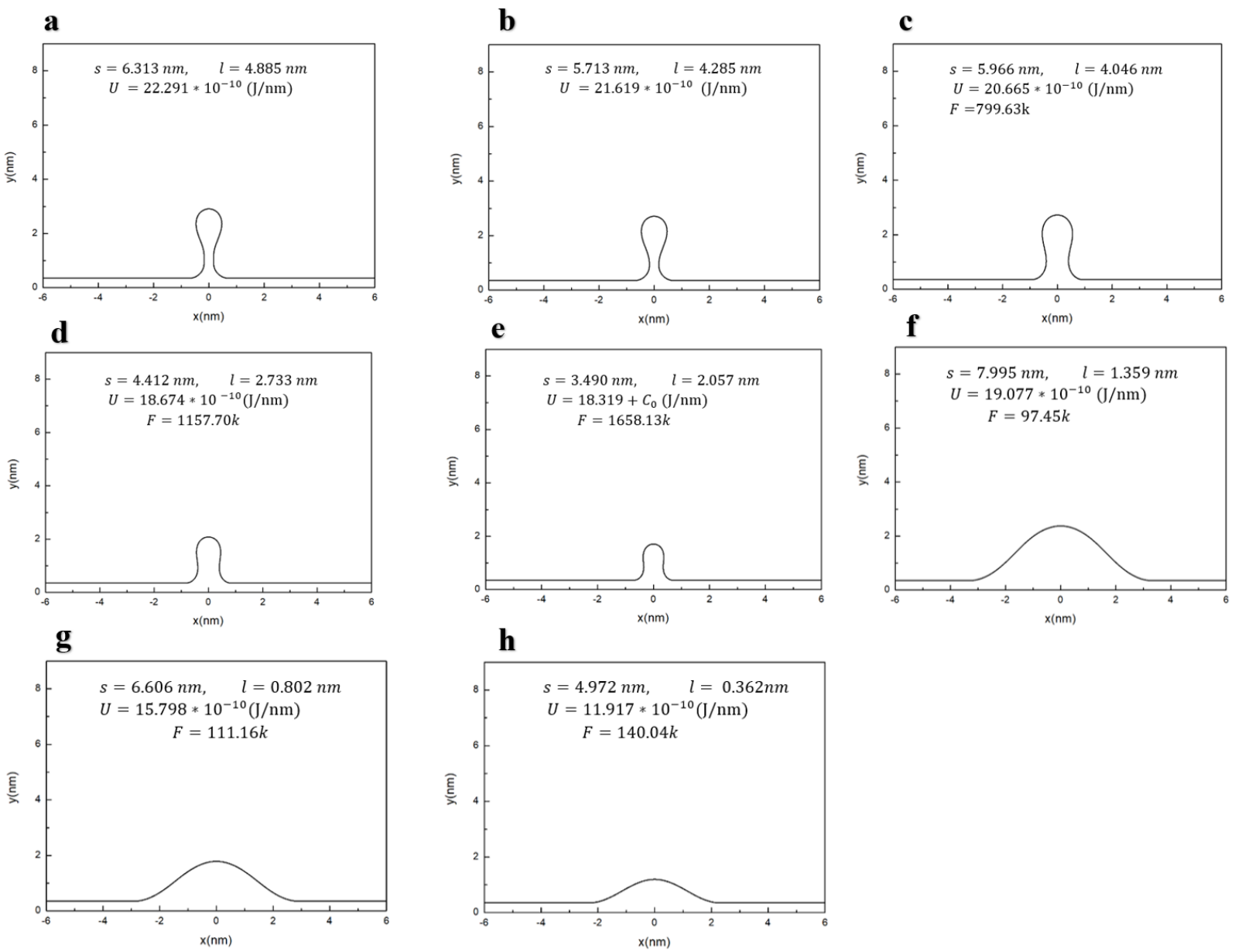

Figure S8| Change in the wrinkle shapes with decreasing lengths in type 3 (a), (b) and type 2 (c)-(e) and type 1 (f)-(h). 
a
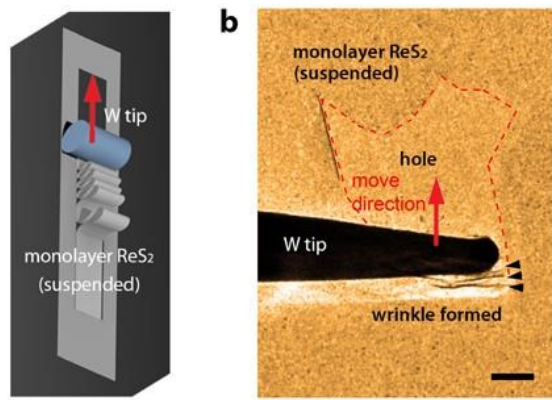

d
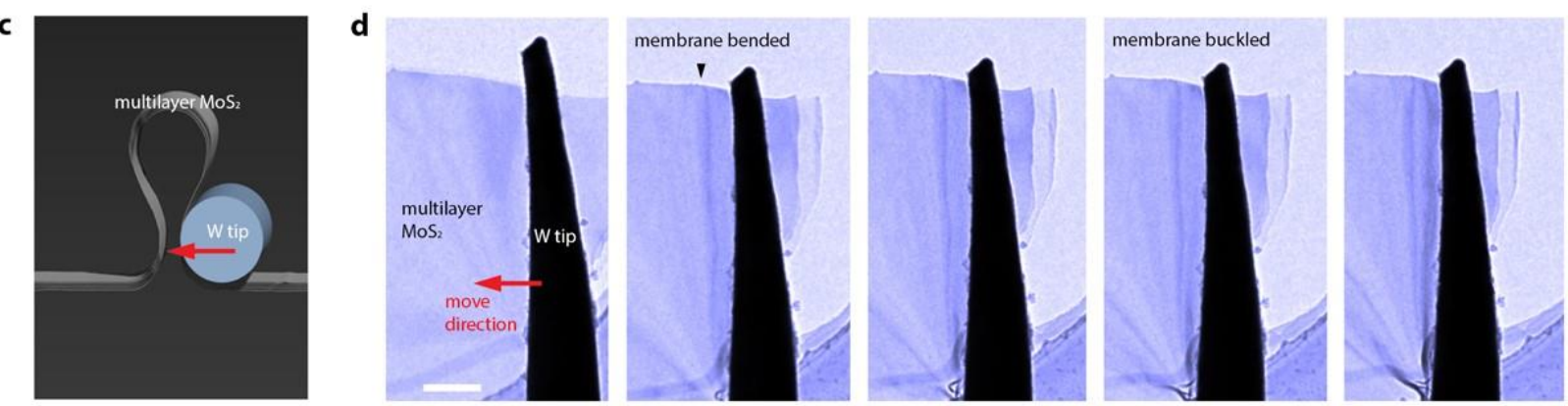

Figure S9| Two-point fixture wrinkle testing on suspended 2D membranes. (a), Scheme for the two-point fixture manipulation on the suspended 2D monolayer $\mathrm{ReS}_{2}$. (b), Serial TEM micrographs for the testing on the monolayer $\mathrm{ReS}_{2}$ sample, scale bar is $100 \mathrm{~nm}$. The move direction of the W tip and wrinkle locations has been highlighted. (c), Scheme for the two-fixture manipulation on the multilayer $\mathrm{MoS}_{2}$. (d), Serial TEM micrographs for the testing on multilayer $\mathrm{MoS}_{2}$, scale bar is $100 \mathrm{~nm}$. 